\title{
Printed Multilayer Superstructures of Aligned Single-Walled Carbon Nanotubes for Electronic Applications
}

2007

Vol. 7, No. 11

$3343-3348$

\author{
Seong Jun Kang, ${ }^{\dagger, £}$ Coskun Kocabas, ${ }^{\ddagger}$ Hoon-Sik Kim, ${ }^{\dagger}$ Qing Cao, ${ }^{\perp}$ \\ Matthew A. Meitl, ${ }^{\dagger}$ Dahl-Young Khang, ${ }^{\dagger}$ and John A. Rogers ${ }^{\star,},+, \neq,,\|\|,, \ldots,+,+$ \\ Department of Materials Science and Engineering, Department of Physics, \\ Department of Mechanical Science and Engineering, Department of Electrical and \\ Computer Engineering, Department of Chemistry, Beckman Institute for Advanced \\ Science and Technology, Frederick Seitz Materials Research Laboratory, University of \\ Illinois, Urbana-Champaign, Illinois, and Division of Advanced Technology, \\ Korea Research Institute of Standards and Science, Daejeon, 305-340 South Korea
}

Received July 3, 2007; Revised Manuscript Received September 18, 2007

\begin{abstract}
We developed means to form multilayer superstructures of large collections of single-walled carbon nanotubes (SWNTs) configured in horizontally aligned arrays, random networks, and complex geometries of arrays and networks on a wide range of substrates. The approach involves guided growth of SWNTs on crystalline and amorphous substrates followed by sequential, multiple step transfer of the resulting collections of tubes to target substrates, such as high-k thin dielectrics on silicon wafers, transparent plates of glass, cylindrical tubes and other curved surfaces, and thin, flexible sheets of plastic. Electrical measurements on dense, bilayer superstructures, including crossbars, random networks, and aligned arrays on networks of SWNTs reveal some important characteristics of representative systems. These and other layouts of SWNTs might find applications not only in electronics but also in areas such as optoelectronics, sensors, nanomechanical systems, and microfluidics.
\end{abstract}

Single-walled carbon nanotubes (SWNTs) might represent an interesting semiconductor material for electronic applications, as suggested by the high current-carrying capacities, ideal subthreshold behavior, and high-field effect mobilities in single tube devices. ${ }^{1-3}$ Although devices of this type can be used to achieve representative demonstrations in electronics, optoelectronics, sensing and other areas, their integration into a scalable technology poses many significant challenges. ${ }^{4-8}$ One approach to avoid these difficulties relies on collections of tubes, either in random, submonolayer networks ${ }^{9-11}$ or, preferably, horizontally aligned arrays, ${ }^{12-16}$ as effective thin film type semiconductors. Films based on arrays can in fact be used to form transistors and simple circuits (i.e., logic gates) with reproducible, device level characteristics that

* To whom correspondence should be addressed. E-mail: jrogers@ uiuc.edu.

Department of Materials Science and Engineering, University of Illinois.

$\doteqdot$ Department of Physics, University of Illinois.

$\S$ Department of Mechanical Science and Engineering, University of Illinois.

"Department of Electrical and Computer Engineering, University of Illinois.

${ }^{\perp}$ Department of Chemistry, University of Illinois.

\# Beckman Institute for Advanced Science and Technology, University of Illinois.

+ Frederick Seitz Materials Research Laboratory, University of Illinois.

${ }^{£}$ Korea Research Institute of Standards and Science. approach expectations based on some of the best single tube devices. ${ }^{12}$ Certain applications, however, require collections and layouts of tubes that are more complex than simple aligned arrays or random networks. Synthetic approaches for such configurations, sometimes in multilayer layouts, are therefore of some interest. Recent reports show that methods based on gas flow, ${ }^{17}$ electrospinning, ${ }^{18}$ and electric fields ${ }^{19,20}$ can provide some useful capabilities. Drawbacks include some combination of moderate to low densities, uncertain uniformity and electrical properties, and complex fabrication processes. In this paper, we present a strategy that involves the guided growth of aligned arrays, random networks and complex combinations of arrays and networks of SWNTs, followed by physical transfer of these tubes, in multiple, sequential steps, to target substrates. This approach enables the formation of large scale multilayer superstructures of complex layouts of SWNTs on substrates ranging from silicon wafers, to flat and curved glass plates to thin plastic sheets. Electrical measurements illustrate some of the properties of several layouts of tubes formed in this manner.

The first step of the process, which is illustrated in its entirety in Figure 1, involves the growth of collections of SWNTs, typically at coverages somewhat less than a 


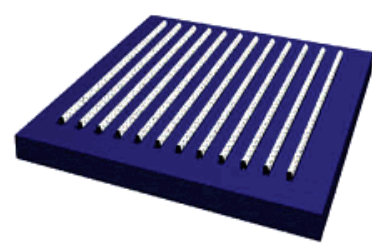

(a) Grow SWNTs

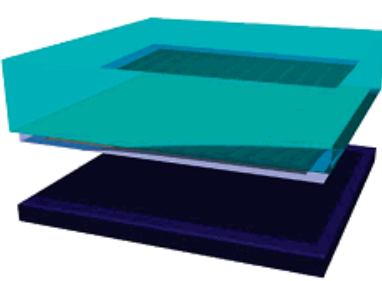

(c) Quickly peel stamp from growth substrate

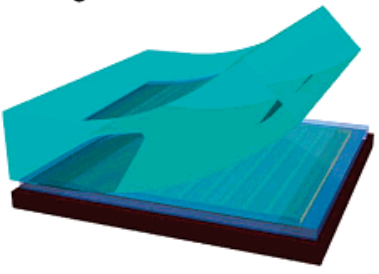

(e) Slowly peel back stamp

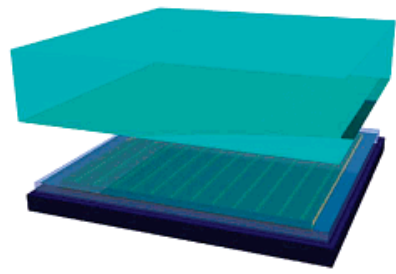

(b) Coat SWNTs with layers of Au \& PI (PVA); apply stamp

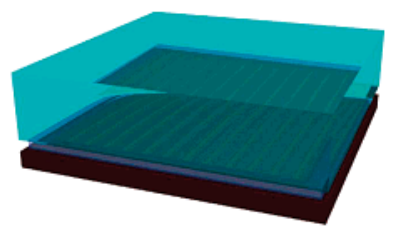

(d) Apply stamp to receiving substrate

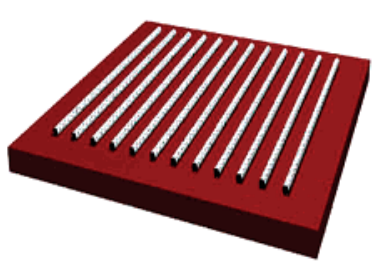

(f) Remove PI (PVA) and Au layers
Figure 1. Schematic illustration of a printing-like process for transferring well-defined collections of SWNTs from a growth substrate to a receiving substrate. The process consists of several steps, beginning with patterned growth of SWNTs (aligned arrays in this case) on a substrate (quartz in this case) by chemical vapor deposition (a), followed by deposition of a bilayer of Au/polymer (either PI or PVA) as a carrier film (b), and removal of the resulting SWNT/Au/polymer layer with an elastomeric stamp (c). Applying the stamp to a receiving substrate (d) and slowing peeling back the stamp leaves the SWNT/Au/polymer on the receiver (e). Finally, removing the polymer and $\mathrm{Au}$ by etching completes the process (f).

monolayer. We used recently reported chemical vapor deposition (CVD) growth techniques with patterned and unpatterned catalyst on either single-crystal quartz substrates for aligned arrays and random networks ${ }^{12-14}$ or amorphous $\mathrm{SiO}_{2} / \mathrm{Si}$ for random networks. ${ }^{21}$ The catalyst consisted of solution cast ferritin (Aldrich; diluted in deoinized (DI) water with concentration $4.25 \mathrm{mg} / \mathrm{mL}$ ) for the random networks and submonolayers of iron $(<0.5 \mathrm{~nm})$ deposited by electron beam evaporation for the aligned arrays. Photolithographic techniques were used to define patterns of both types of catalyst, using procedures described elsewhere. ${ }^{12,14}$ For all cases, the CVD growth used a flow of argon $(10 \mathrm{sccm})$ and hydrogen $(10 \mathrm{sccm})$ through an ethanol bubbler and a temperature of $925^{\circ} \mathrm{C}$. By combined control of the catalyst layout, the substrate type and the growth conditions, configurations of tubes ranging from random networks to perfectly aligned arrays and anything in between as well as integrated layouts of electrically connected random networks and aligned arrays in various geometries, are possible. ${ }^{12-15}$

The transfer process moves these collections of tubes from the growth substrate to another surface, without changing their coverage, geometry, or other properties of their layouts.
The general strategy involves first the deposition of a thin layer of solid material, which we refer to as the "carrier", on top of the SWNTs. Next, a kinetically controlled printing process $^{22}$ with a soft, elastomeric stamp peels this film from the growth substrate and delivers it to a target substrate. Etching the layers off of the tubes completes the process. The materials for the carrier film are critically important; they must (i) effectively encapsulate the exposed parts of the SWNTs to enable their efficient removal from the growth substrate, (ii) provide a mechanically strong structural element for peel-back, and (iii) remove easily from the SWNTs after transfer in a manner that does not degrade the properties of the SWNTs. We find that a bilayer of Au and either polyimide (PI) or polyvinyl alcohol (PVA) provides all of these required characteristics. The polymer provides physical toughness for clean separation. The metal can be removed from the SWNTs by wet chemical etching without significantly altering their properties or layouts. These selections, together with the kinetically controlled printing approach, which obviates the need for an etching step to lift off the SWNTs from their growth substrate, represent important differences between the transfer techniques described here and those previously reported. ${ }^{21}$ These differences lead to the ability to manipulate SWNT arrays, integrated arrays, and networks as well as multilayer stacks in ways that were previously impossible.

Figure 1 schematically illustrates the transfer process. The first step involved deposition of a thin layer of $\mathrm{Au}(\sim 100$ $\mathrm{nm})$ by electron beam evaporation $\left(3 \times 10^{-6}\right.$ Torr; Temescal CV-8) onto the SWNTs. Spin casting (3000 rpm for $30 \mathrm{~s}$ ) formed a partially cured film of polyamic acid (PI; polyamic acid, Aldrich) on top of this Au layer; heating at $110^{\circ} \mathrm{C}$ for 2 min removed the solvent and partially cured the PI. A flat slab of poly(dimethylsiloxane) (PDMS; Sylgard 184, Dow Corning) elastomer provided the stamp. Placing this stamp in conformal contact with the PI/Au/SWNTs/substrate and then quickly peeling it away lifts the film of PI/Au/SWNTs off of the substrate and leaves it van der Waals adhered to the surface of the stamp (Figure 1c). The stamp with the layers of PI/Au/SWNTs was then placed on a receiving substrate. Slowly peeling back the PDMS leaves the PI/Au/ SWNT on this receiving substrate thereby completing the transfer. The PI/Au carrier was removed by oxygen reactive ion etching (RIE) (150 mtorr, $20 \mathrm{sccm} \mathrm{O}_{2}, 150 \mathrm{~W}, 35 \mathrm{~min}$ ) to eliminate the PI, followed by wet etching with a commercial solution (Au-TFA, Transene) to eliminate the $\mathrm{Au}$. The process with PVA is similar except that the PVA is dried $70{ }^{\circ} \mathrm{C}$ for $30 \mathrm{~min}$ (instead of cured) after spin casting (speed, time, thickness) from a DI water solution. The PVA can be removed by 10 min of rinsing with DI water.

Figure 2a shows a scanning electron microscope (SEM) image of arrays of SWNTs grown on quartz and transferred to $100 \mathrm{~nm} \mathrm{SiO} / \mathrm{Si}$. The inset provides an SEM image of the SWNT arrays on the growth substrate, collected before the transfer process. The bright stripes in these images correspond to the regions of patterned iron catalyst where dense random networks of SWNTs form. As the SWNTs emerge 


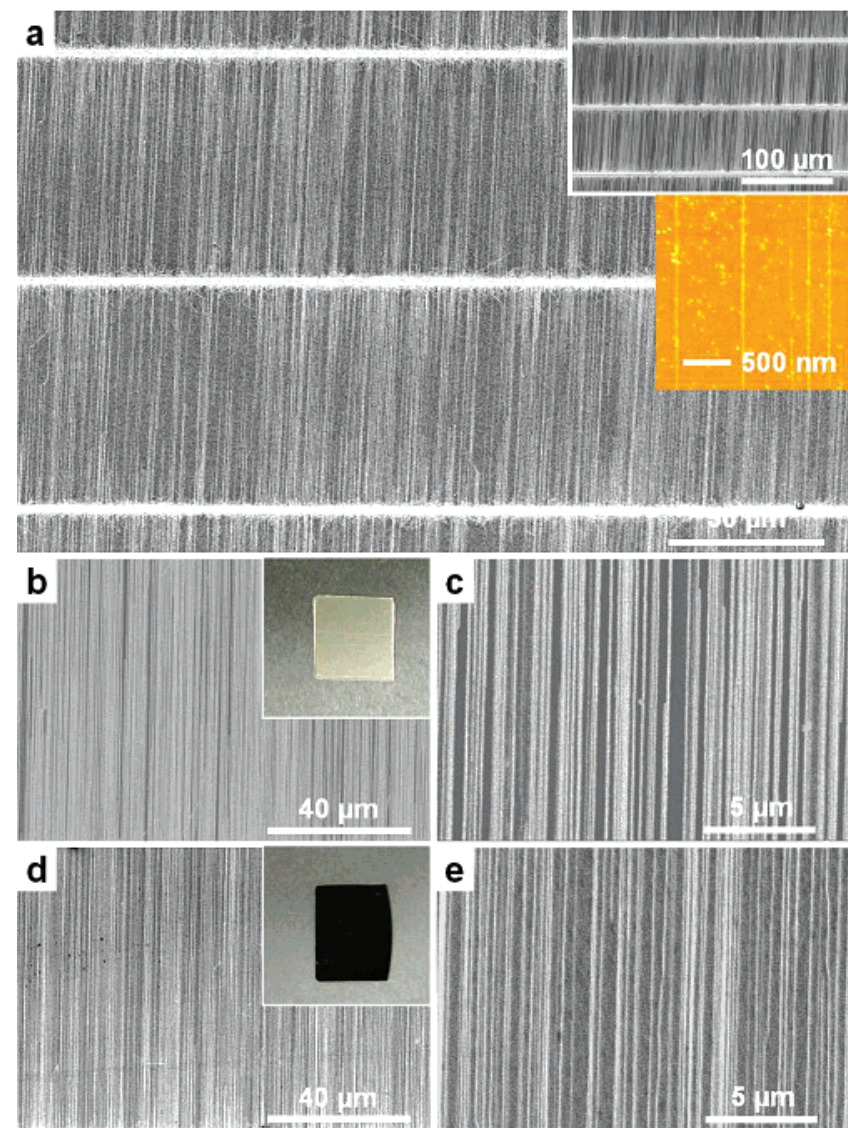

Figure 2. (a) SEMs of aligned arrays of SWNTs transferred to a substrate of $\mathrm{SiO}_{2}(100 \mathrm{~nm}) / \mathrm{Si}$. The inset shows an image of the SWNTs on quartz growth substrate before transfer. The inset AFM shows SWNTs on $\mathrm{SiO}_{2}(100 \mathrm{~nm}) / \mathrm{Si}$ after transfer. (b,c) SEMs of arrays of SWNTs on a quartz growth substrate. (d,e) SEMs of arrays of SWNTs transferred to a receiving substrate of $\mathrm{HfO}_{2}(10 \mathrm{~nm}) / \mathrm{Si}$. These images indicate that the alignment and density of the arrays are not changed substantially by the transfer process.

from these regions onto the bare quartz, they align with the [211 10 ] direction. ${ }^{13}$ Figure $2 \mathrm{~b}, \mathrm{c}, \mathrm{d}$,e shows high-magnification SEM images of the SWNTs arrays on quartz before transfer and after transfer to a thin $(10 \mathrm{~nm})$ layer of $\mathrm{HfO}_{2}$ substrate on $\mathrm{Si}$, respectively. As indicated by these images, the density, alignment, linearity, and other features of the SWNT arrays are unaltered by the process. The atomic force microscope (AFM) image in Figure 2a shows nanotube arrays after transfer on $100 \mathrm{~nm} \mathrm{SiO} / 2 / \mathrm{Si}$ and a short exposure to $\mathrm{H}_{2} \mathrm{O}_{2}$ : $\mathrm{H}_{2} \mathrm{SO}_{4}=2: 1$ to eliminate residual byproducts from RIE of the PI. This image indicates that only a small amount of residue remains, such that the arrays of SWNTs can be seen clearly. Improvements in the processing might lead to even smaller residue, for applications that are critically sensitive.

This same procedure is compatible with random networks and other layouts of tubes. For example, patterning the catalyst into complex shapes on quartz leads to random networks of SWNTs in these regions and aligned arrays in the other regions. Figure 3a,b shows an example where the catalyst is in the geometry of a ring oscillator. The bright areas correspond to dense networks of tubes; these regions are interconnected by aligned arrays. Figure $3 \mathrm{c}, \mathrm{d}$ presents the same type of structure after transfer to $\mathrm{SiO}_{2}(100 \mathrm{~nm}) / \mathrm{Si}$.
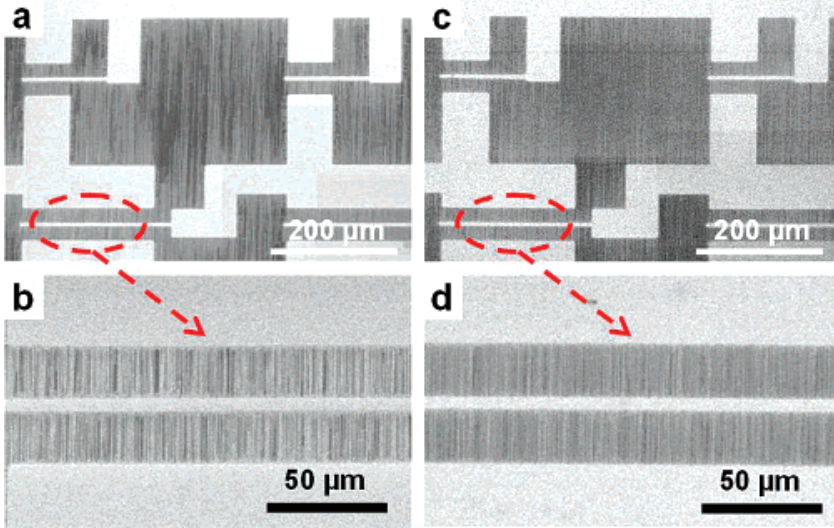

Figure 3. SEMs of complex patterns of dense, random networks of SWNTs (bright regions) interconnected by aligned arrays of SWNTs, as grown on quartz (a,b) and transferred to $\mathrm{SiO}_{2}(100 \mathrm{~nm}) /$ Si $(c, d)$.
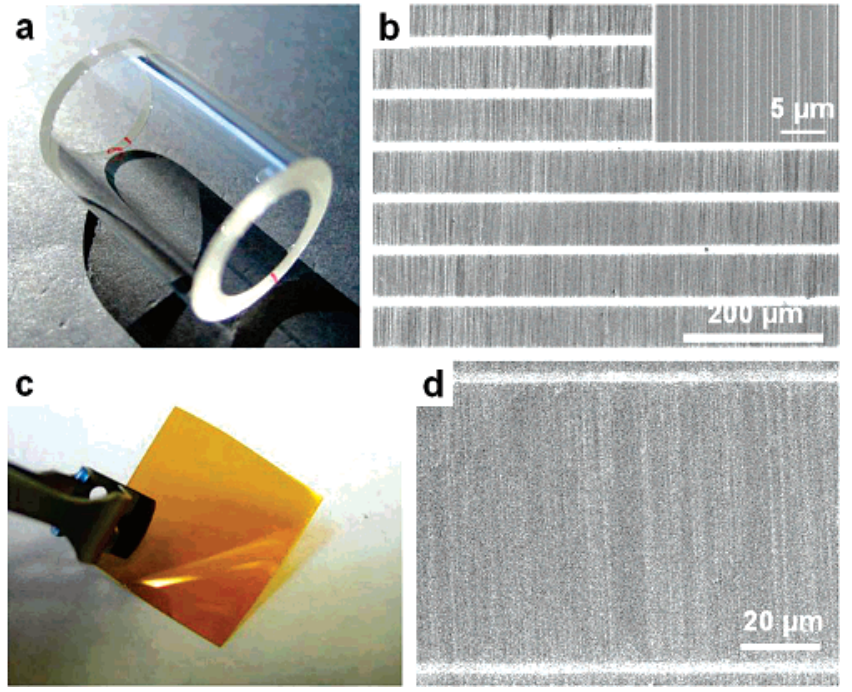

Figure 4. Optical image (a) and SEM (b) of aligned arrays of SWNTs transferred from a quartz growth substrate to the surface of a glass cylinder. The inset in (b) provides a high-magnification view. Optical image (c) and SEM (d) of aligned arrays of SWNTs transferred from a quartz growth substrate to a thin sheet of polyimide (Kapton).

The efficiency in this case is similar to the simpler structures of aligned arrays. The same procedures can be used with other substrates, provided that they are sufficiently smooth to receive the $\mathrm{Au} / \mathrm{PI} / \mathrm{SWNT}$ layer. Figure 4a,c shows, as examples, SWNT arrays transferred to the curved surface of a glass tube and a thin sheet of plastic (Kapton, DuPont). These results suggest that high-quality SWNT arrays can be integrated with wide ranging classes of substrates, which are important for imaging systems, flexible electronics, and other applications.

An important aspect of the approach is its ability to transfer tubes onto substrates that already support tubes and can yield well-defined multilayer superstructures of SWNTs. For example, bilayer crossbar arrays can be formed by transferring an aligned array on top of another array with a rotation of ninety degrees, as shown in Figure 5a. The bright crossed lines correspond to the regions of catalyst in the two arrays. 


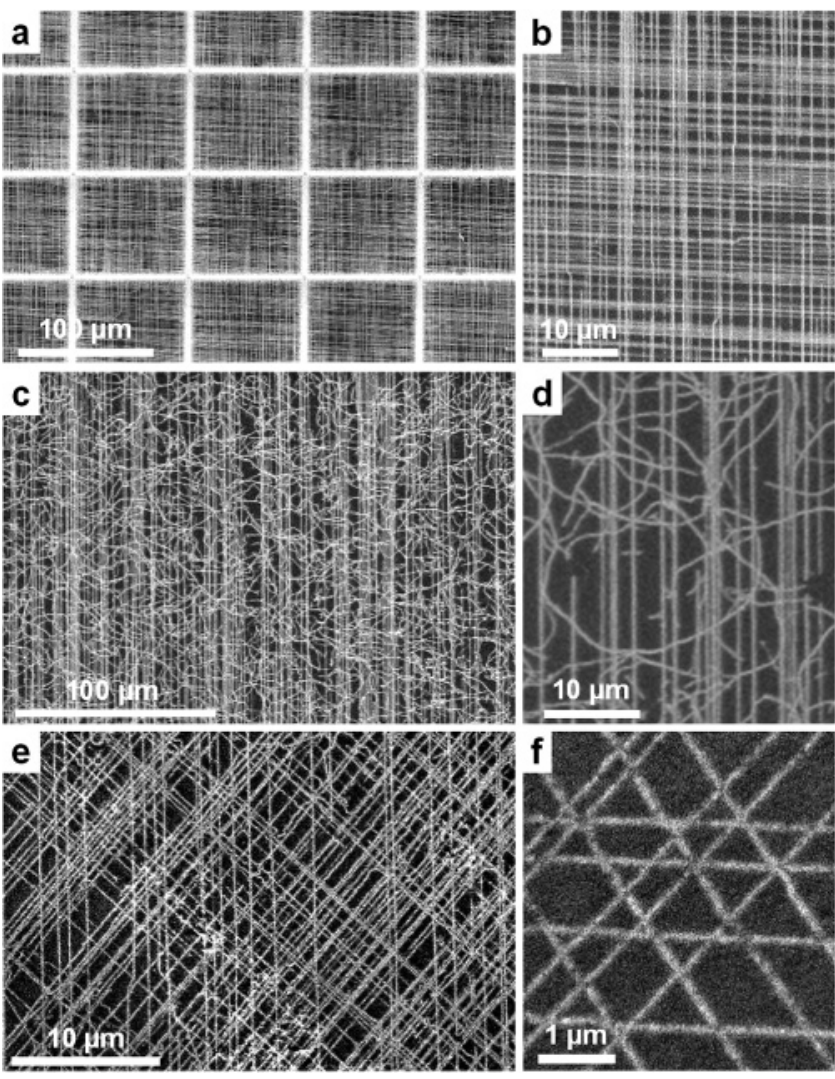

Figure 5. SEMs of various types of multilayer stacks of SWNTs formed by transfer. (a,b) SWNTs in the geometry of crossbar arrays formed by a transfer process. (c,d) Random network of SWNTs transferred on top of an aligned array. (e,f) SWNTs in the geometry of a triangle lattice, formed by a two-step transfer process.

Figure $5 \mathrm{~b}$ illustrates that crossbar arrays of SWNTs, in the form of square mesh structures, exist inside each box defined by these catalyst lines. Transfer of random networks onto aligned arrays produces bilayer structures (Figure $5 c, d$ ) with levels of in-plane anisotropy that can be defined by controlling the coverages of the random network and aligned array components. Similar procedures can be repeated to yield additional layers. For example, two transfer steps onto a target substrate with aligned arrays, using rotation angles of 60 degrees, forms trilayer, triangular mesh structures, as shown in Figure 5e,f.

An obvious potential area of application of these structures of SWNTs is in electronics, either as field effect transistors, transparent conductors, or related elements. For these and other applications, the ability to pattern electrodes, dielectrics, and other materials by photolithography is important. We found that even in these multilayer configurations, the SWNTs are sufficiently adherent to the substrate to allow for spin casting of photoresist, patterned exposure, development and resist removal in a lift-off process that defines metal electrodes without substantially altering the coverage or arrangements of the SWNTs. To demonstrate this capability and to evaluate electrical properties of a representative multilayer SWNT structures, we formed collections of electrodes on the aligned arrays (a), the bilayer crossbar layouts (b), and the random networks (c) as shown in Figure 6. In this process, photolithography defined openings in a (a)
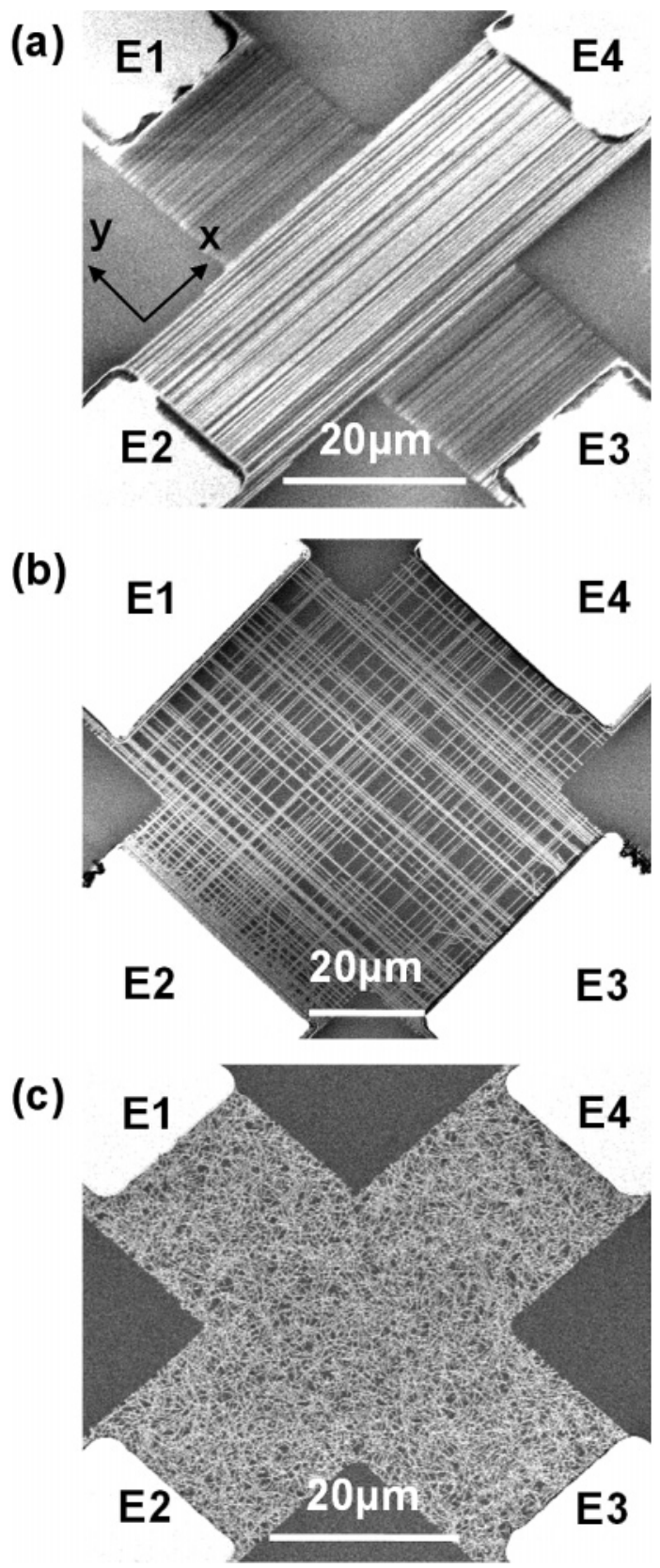

Figure 6. SEMs of four terminal test structures formed on SWNTs with different layouts, including (a) aligned arrays, (b) crossbars, and (c) random networks.

spin cast layer of photoresist (AZ 5214; $1.6 \mu \mathrm{m}$ at $3000 \mathrm{rpm}$, Clariant). Blanket deposition of a bilayer of $\mathrm{Ti}(1 \mathrm{~nm}) / \mathrm{Pd}$ $(20 \mathrm{~nm})$ by electron beam evaporation $\left(3 \times 10^{-6}\right.$ Torr; Temescal CV-8) followed by removal of the resist with acetone defined the electrodes. The geometry consisted of arrays of devices, each with four pads either $40 \times 40$ or 20 $\times 20 \mu \mathrm{m}$ in size, separated by $60 \mu \mathrm{m}$. To isolate these devices, the region between the electrodes was first protected by a patterned layer of photoresist (AZ 5214; $1.6 \mu \mathrm{m}$ at $3000 \mathrm{rpm}$, Clariant) aligned to the devices. Reactive ion etching (50 mtorr, $20 \mathrm{sccm} \mathrm{O}, 100 \mathrm{~W}, 30 \mathrm{~s}$ ) then removed the exposed SWNTs, and a rinse in acetone washed away the remaining resist. Figure 6 presents images of the types of devices that were investigated. Current-voltage characteristics were measured in each case with various probing 
(a)

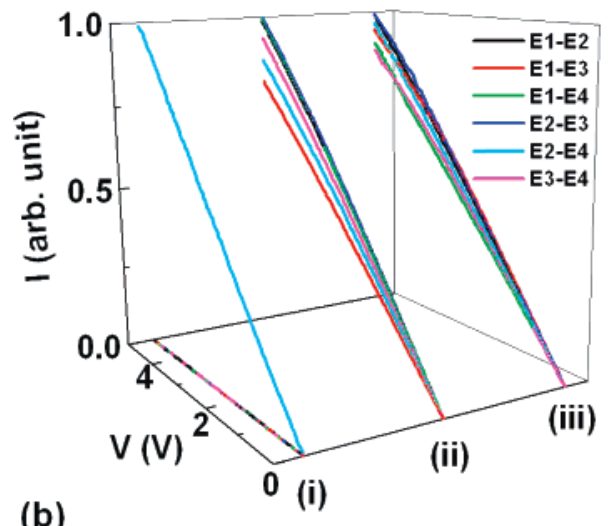

(b)

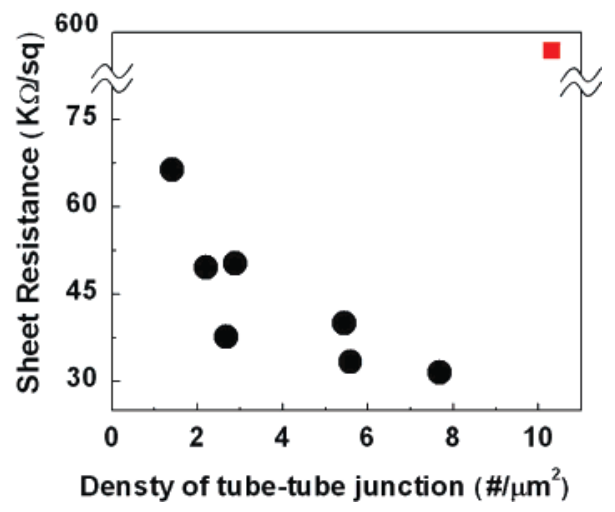

Figure 7. (a) Electrical measurements on different types of "films" of SWNT generated by growth and transfer, including aligned arrays (i), crossbars (ii), and random networks (iii) with various four terminal probing combinations (E1-E2, E1-E3, E1-E4, E2-E3, E2$\mathrm{E} 4, \mathrm{E} 3-\mathrm{E} 4)$. The graph shows current $(I)$ and voltage $(V)$ response that indicates linear, metallic response with levels of anisotropy that reflect the underlying anisotropy of the SWNT arrangements in the films. Panel (b) shows sheet resistances of crossbar layouts of SWNTs (black symbols) as a function of the density of tubetube junctions. The red square corresponds to the case of a random network of SWNTs.

combinations using different pairs of electrodes (E1, E2, E3, and E4). As shown in Figure 7a, the device with aligned arrays showed extremely strong anisotropy in the properties. In particular, current flowed only between electrodes E2 and E4; other probing combinations revealed currents at the noise limit of our setup. In the case of other devices (Figure 6b,c), current was observed between all pairs of electrodes, due to the possibility of percolation pathways associated with tube/ tube junctions. Using a definition of the anisotropy $(A)$ according to

$$
A=\left|\frac{I_{x}-I_{y}}{I_{x}+I_{y}}\right|
$$

$I_{x}$ is the current between E2 and E4, while $I_{y}$ is the current between $\mathrm{E} 1$ and $\mathrm{E} 3$. We find that the aligned arrays have $A$ $=1$ (Figure 6a), the crossbar layouts have $A=0.04$ (Figure $6 \mathrm{~b}$, which has a 105 tubes in $x$-direction while 85 tubes in $y$-direction), and the single layer random networks have $A$ $=0.01$ (Figure 6c). Moreover, if we change the number of tubes in each direction of crossbar layout, we can obtain various films with different anisotropy factors. For example, we obtained $A=0.14$ (47 tubes in $x$-direction and 91 tubes in $y$-direction) and $A=0.08$ (75 tubes in $x$-direction and 116 tubes in $y$-direction). These results demonstrate some flexibility in the levels of anisotropy that can be engineered into films of SWNTs by use of transfer approaches. Such layouts could be useful for various applications in chemical sensing, transparent electronics, light-emitting devices, logic circuits, and memory cells.

Next, we used these types of devices to determine, using the four-point probe method (Van der Pauw formula) ${ }^{23}$ sheet resistances from the measured resistances between pairs of electrodes for crossbar arrays with different densities of tube/ tube junctions, $D$. In particular, constant current was applied to E1 and E2 (and, separately, E2-E3, E3-E4, and E4-E1), while the voltage was measured between E3 and E4 (and, separately, E4-E1, E1-E2, and E2-E3). Sheet resistance of the film can be written ${ }^{24}$ as $R_{\mathrm{S}}=C(\lambda) R$, where $C(\lambda)$ is a correction coefficient which is derived as $C(\lambda)=\pi / \ln 2+$ $(1 / 64)\left(\pi^{3} /(\ln 2)^{2}\right) \lambda^{2},{ }^{24}$ and $\lambda$ is the ratio of contact length to total length of the boundary of the sheet.

$$
\begin{gathered}
e^{-\pi R_{\text {vertical }} / R}+e^{-\pi R_{\text {horizontal }} / R}=1 \\
R_{\text {vertical }}=\frac{R_{\mathrm{E} 1-\mathrm{E} 2, \mathrm{E} 3-\mathrm{E} 4}+R_{\mathrm{E} 3-\mathrm{E} 4, \mathrm{E} 1-\mathrm{E} 2}}{2} \\
R_{\text {horizontal }}=\frac{R_{\mathrm{E} 2-\mathrm{E} 3, \mathrm{E} 4-\mathrm{E} 1}+R_{\mathrm{E} 4-\mathrm{E} 1, \mathrm{E} 2-\mathrm{E} 3}}{2} \\
R_{\mathrm{E} 1-\mathrm{E} 2, \mathrm{E} 3-\mathrm{E} 4}=\frac{V_{\mathrm{E} 3-\mathrm{E} 4}}{I_{\mathrm{E} 1-\mathrm{E} 2}}
\end{gathered}
$$

Here, vertical and horizontal refer to the $\hat{x}+\hat{y}$ and $\hat{x}-\hat{y}$ directions, respectively. For the device geometry shown in Figure $6 \mathrm{~b}, \lambda \sim 0.7$, which provides a correction coefficient of $\sim 5$. Figure $7 \mathrm{~b}$ shows the results. The sheet resistances varied from $\sim 66.5 \mathrm{k} \Omega / \mathrm{sq}$ at $D=\sim 1.4$ juntions $/ \mu \mathrm{m}^{2}$ to $\sim 31.5$ $\mathrm{k} \Omega / \mathrm{sq}$ at $D=\sim 7.7$ juntions $/ \mu \mathrm{m}^{2}$. The sheet resistance of the random network in Figure $6 \mathrm{c}$ is $\sim 585 \mathrm{k} \Omega / \mathrm{sq}$ at $D=$ $\sim 10$ juntions $/ \mu \mathrm{m}^{2}$. The silicon substrate was grounded during the measurement. The two-terminal (E2-E4) resistance of the aligned nanotube film (Figure 6a) is $\sim 50 \mathrm{k} \Omega$ (surface coverage: $\sim 0.5 \%$ ), while the value is $\sim 495 \mathrm{k} \Omega$ for the random network nanotube film as shown in Figure $6 \mathrm{c}$ (surface coverage: $\sim 1.1 \%$ ).

In summary, we developed a method for transferring and combining collections of SWNTs in a wide variety of formats ranging from random networks to perfectly aligned arrays, and integrated versions of these geometries. We demonstrated SWNTs arrays, formed by guided growth on single-crystal quartz substrates, to other surfaces including glass, plastic, and high k-films without substantially altering the alignment or coverage of the SWNTs. Simple structures, including transparent conductors, demonstrate the electrical functionality of single and multilayer SWNT layouts. These results might be of interest for various applications of SWNTs in electronics, optoelectronics, sensors, and nanomechanical systems. 
Acknowledgment. We thank T. Banks and K. Colravy for help with processing using facilities at the Frederick Seitz Materials Research Laboratory. This material is based upon work supported by the National Science Foundation under Grant NIRT-0403489 and the U.S. Department of Energy, Division of Materials Sciences under Award No. DEFG0291ER45439, through the Frederick Seitz MRL and Center for Microanalysis of Materials at the University of Illinois at Urbana-Champaign. S.J.K. acknowledges fellowship support from the Institute of Information Technology Assessment of Korea.

Supporting Information Available: Layout of the SWNT TFT device and transfer curve of the device. This material is available free of charge via the Internet at http:// pubs.acs.org.

\section{References}

(1) Yao, Z.; Kane, C. L.; Dekker, C. Phys. Rev. Lett. 2000, 84, 2941.

(2) Lin, Y.-M.; Appenzeller, J.; Knoch, J.; Avouris, P. IEEE Trans. Nanotechnol. 2005, 4, 481.

(3) Durkop, T.; Getty, S. A.; Cobas, E.; Fuhrer, M. S. Nano Lett. 2004, 4,35 .

(4) Chen, Z.; Appenzeller, J.; Lin, Y.-M.; Sippel-Oakley, J.; Rinzler, A. G.; Tang, J.; Wind, S. J.; Solomon, P. M.; Avouris, P. Science 2006, $311,1735$.

(5) Bachtold, A.; Hadley, P.; Nakanishi, T.; Dekker, C. Science 2001, 294, 1317.

(6) Misewich, J. A.; Martel, R.; Avouris, P.; Tsang, J. C.; Heinze, S.; Tersoff, J. Science 2003, 300, 783.
(7) Chen, J.; Perebeinos, V.; Freitag, M.; Tsang, J.; Fu, Q.; Liu, J.; Avouris, P. Science 2005, 310, 1171

(8) Kong, J.; Franklin, N. R.; Zhou, C.; Chapline, M. G.; Peng, S.; Cho, K.; Dai, H. Science 2000, 287, 622.

(9) Cao, Q.; Xia, M.-G.; Shim, M.; Rogers, J. A. Adv. Func. Mater. 2006, 16, 2355.

(10) Artukovic, E.; Kaempgen, M.; Hecht, D. S.; Roth, S.; Gruner, G. Nano Lett. 2005, 5, 757.

(11) Snow, E. S.; Perkins, F. K. Nano Lett. 2005, 5, 2414.

(12) Kang, S. J.; Kocabas, C.; Ozel, T.; Shim, M.; Pimparkar, N.; Alam, M. A.; Rotkin, S. V.; Rogers, J. A. Nat. Nanotechnol. 2007, 2, 230.

(13) Kocabas, C.; Hur, S.-H.; Gaur, A.; Meitl, M. A.; Shim, M.; Rogers, J. A. Small 2005, 1, 1110 .

(14) Kocabas, C.; Shim, M.; Rogers, J. A. J. Am. Chem. Soc. 2006, 128 , 4540.

(15) Kocabas, C.; Pimparkar, N.; Yesilyurt, O.; Kang, S. J.; Alam, M. A.; Rogers, J. A. Nano Lett. 2007, 7, 1195.

(16) Liu, X.; Han, S.; Zhou, C. Nano Lett. 2006, 6, 34.

(17) Huang, S.; Maynor, B.; Cai, X.; Liu, L. Adv. Mater. 2003, 15, 1651.

(18) Gao, J.; Yu, A.; Itkis, M. E.; Bekyarova, E.; Zhao, B.; Niyogi, S.; Haddon, R. C. J. Am. Chem. Soc. 2004, 126, 16698

(19) Ismach, A.; Joselevich, E. Nano Lett. 2006, 6, 1706.

(20) Diehl, M. R.; Yaliraki, S. N.; Beckman, R. A.; Barahona, M.; Heath, J. R. Angew. Chem., Int. Ed. 2002, 41, 353.

(21) Hur, S.-H.; Kocabas, C.; Gaur, A.; Park. O. O.; Shim, M.; Rogers, J. A. J. Appl. Phys. 2005, 98, 114302.

(22) Meitl, M. A.; Zhu, Z.-T.; Kumar, V.; Lee, K. J.; Feng, X.; Huang, Y. Y.; Adesida, I.; Nuzzo, R. G.; Rogers, J. A. Nat. Mater. 2006, 5, 33.

(23) van der Pauw, L. J. Philips Res. Rep. 1958, 13, 1.

(24) Versnel, W. Solid-State Electron. 1979, 22, 911.

NL071596S 